\title{
Monitoring sputum eosinophils in mucosal inflammation and remodelling: a pilot study
}

\author{
J. Chakir*, L. Loubaki*, M. Laviolette*, J. Milot*, S. Biardel*, L. Jayaram*, \\ M. Pizzichini ${ }^{\ddagger}$, E. Pizzichini ${ }^{\dagger}$, F.E. Hargreave ${ }^{\#}$, P. Nair ${ }^{\#}$ and L-P. Boulet*
}

ABSTRACT: Normalisation of eosinophil counts in sputum of asthmatic patients reduces eosinophilic exacerbations. However, the effect of this strategy on airway remodelling remains to be determined. We compared bronchial inflammation and collagen deposition after 2 yrs of treatment guided by either sputum eosinophils (sputum strategy, SS) or by clinical criteria (clinical strategy, CS).

As a pilot study, 20 mild asthmatic patients were randomly assigned to CS or SS strategies. Bronchial biopsies were obtained when minimum treatment needed to maintain control was identified and this was continued for 2 yrs. Biopsies were immunostained for inflammatory cells, mucin 5A (MUC5A) and collagen.

The mean dose of inhaled corticosteroids at the start and end of the study was similar in both SS and CS groups. Forced expiratory volume in $1 \mathrm{~s}$ increased in both groups at the study end. In SS, mucosal lymphocyte and eosinophil counts, but not neutrophils, were reduced at the end of the study. In CS, only activated eosinophil and neutrophil counts decreased. MUC5A staining decreased in SS but not CS. No change in collagen deposition underneath the basement membrane was observed in either strategy.

Treatment strategies that normalise sputum eosinophils also reduce mucosal inflammatory cells and MUC5A expression, but do not change subepithelial collagen deposition in mild to moderate asthma.

KEYWORDS: Airway remodelling, asthma, bronchial biopsies, sputum cell count

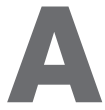
sthma is characterised by variable flow limitation and airway hyperresponsiveness (AHR) caused by airway inflammation and structural changes [1]. Bronchial biopsies have been used to evaluate the airway wall inflammatory and remodelling processes, and guidelines have been published on how this assessment tool can be safely used [2, 3]. Although the effects of specific medications have been studied [4-6], few studies have looked at the influence of various long-term treatment strategies on airway biopsies. SONT et al. [7] and WARD et al. [8] showed that high doses of inhaled corticosteroids (ICS) aimed at reducing AHR were associated with a reduction of subepithelial fibrosis after a 2-yr treatment period.

Although asthma control is usually evaluated by clinical features and expiratory flows, recent observations suggest that monitoring airway inflammation by noninvasive measures such as quantitative sputum cell counts results in a reduction in asthma exacerbations [9-11]. We recently conducted a study to investigate the effect of assessing asthma control and treatment needs using quantitative sputum cell counts. In this study, monitoring sputum cell counts reduced the number of eosinophilic exacerbations without increasing the total corticosteroid dose [11]. As part of this investigation, we undertook a pilot study to look at the effect of treatment strategies based on sputum eosinophil count or clinical evaluation on airway wall inflammation and remodelling in bronchial biopsies over a 2-yr period. The expression of mucin 5A (MUC5A), the principal mucin produced by bronchial epithelial cells, and the thickness of collagen deposited below the epithelium were selected for the latter component of the investigation.

\section{METHODS}

\section{Selection and evaluation of subjects}

20 subjects volunteered for this study (table 1 ). At entry, asthma was confirmed by improvement in forced expiratory volume in $1 \mathrm{~s}$ (FEV1) $\geqslant 12 \%$ after salbutamol $200 \mu \mathrm{g}$, or by the demonstration of AHR to methacholine (provocative concentration of methacholine needed to cause a $20 \%$ fall in FEV1 $<8 \mathrm{mg} \cdot \mathrm{mL}^{-1}$ ). All subjects were either nonsmokers

\section{AFFILIATIONS}

*Centre de recherche de l'Hôpital

Laval, Institut universitaire de

cardiologie et de pneumologie,

Sainte-Foy, QC,

\#Firestone Institute for Respiratory Health, St. Joseph's Healthcare and McMaster University, Hamilton, ON, Canada, and

- NuPAIVA-Asthma Research Center. Florianopolis, Brazil.

\section{CORRESPONDENCE}

J. Chakir

Hôpital Laval

2725 Chemin Ste-Foy

Sainte-Foy

G1V 4G5

QC

Canada

E-mail: jamila.chakir@med.ulaval.ca

Received:

Aug 222008

Accepted after revision:

July 042009

First published online:

July 162009 
or ex-smokers of $<10$ pack-yrs for $>6$ months. None had other chronic respiratory disease, a history of respiratory infection within the past 2 months, or other uncontrolled systemic or psychiatric diseases. The study was approved by the Laval Hospital (Sainte-Foy, QC, Canada) ethics committee and all subjects signed written informed consent.

\section{Study design}

The subjects were from one (Laval Hospital) of the four centres participating in a randomised controlled trial to investigate the effectiveness of using quantitative sputum cell counts (induced or spontaneous) to guide corticosteroid and antibiotic treatment [11]. These subjects were comparable to the entire cohort in terms of demographic characteristics and sputum cell counts, but had less airflow obstruction. Subjects randomised at Laval to the clinical strategy (CS) also were less hyperresponsive to methacholine compared with the entire cohort and on smaller daily dose of ICS. The subjects were randomised to treatment guided by symptoms and spirometry according to Canadian Consensus Guidelines [12] (CS), or by sputum cell counts to keep eosinophils within the normal range of $\leqslant 2.0 \%$ (sputum strategy (SS)). In both strategies, the subjects were blind to the strategy allocation and to sputum cell counts. In the CS, the investigators were blind to the sputum cell counts.

In the first phase of the study, the dose of ICS was adjusted to identify the minimum needed to maintain asthma control for 1 month. In the second phase, this minimum treatment was maintained and the subjects were seen every 3 months for 24 months from the start of the study. Adjustments to treatment were made in both phases if there was clinical deterioration and, in the SS group, if sputum eosinophils (assessed every 3 months) increased over 2\%. Bronchial biopsies were obtained at the end of both phases.

\section{Procedures}

Sputum induction and processing for total and differential cell counts were performed by the methods described by PIZZICHINI et al. [13]. Bronchoscopies were performed as previously described [6]. The biopsies were embedded in glycolmethacrylate monomer (Polyscience, Warrington, PA, USA) and immunostained as previously described [14] with the following antibodies: mouse anti-human CD3, and CD4 for Tlymphocytes, tryptase for mast cells, neutrophil-elastase (Dako Diagnostics, Missisauga, ON, Canada), and EG1 and EG2 (Kabi Pharmacia Diagnostics, Baie D’Urfay, QC, Canada), mouse anti-human mucin 5A (Abcam, Cambridge, MA, USA) and mouse anti-human type-I collagen and mouse anti-human type-III collagen monoclonal antibodies (MediCorp Inc., Montreal, QC, Canada). All slides were coded and sections counted blindly. Sections obtained at the end of phase 1 and 2 were processed together to maintain identical conditions. Counts were expressed as number of positive cells per $\mathrm{mm}^{2}$ of bronchial submucosa, excluding mucus glands, blood vessels and smooth muscles. The mean intra-observer variability (three repeated cell counts) was $4-8 \%$ for all studied antibodies. Mucin 5A staining quantification was performed using Image Pro-Plus software (Media Cybernetics, Bethesda, MD, USA). Quantification was expressed as a percentage of staining intensity per $\mathrm{mm}^{2}$ using colour segmentation as previously reported $[15,16]$. The thickness of the collagen layer below the basement membrane was quantified using Image Pro-Plus software. These data are expressed as the mean of three measurements.

\section{Statistical analysis}

Clinical characteristic data were analysed using t-tests or the Wilcoxon rank sum test for continuous variables and the Fisher's exact test for categorical variables. Values from bronchial biopsy immunostaining were log transformed to stabilise variances. The crossed-nested design was involved to analyse three experimental factors: one associated to the comparison between two clinical strategies, factor strategy (fixed); one linked to the subjects, nested random factor in strategy group; and one associated to the comparison between results at baseline and 2 yrs, factor period (fixed). The factor period was analysed as a repeated factor. A mixed model

TABLE 1 Clinical characteristics at baseline and the end of phases 1 and 2

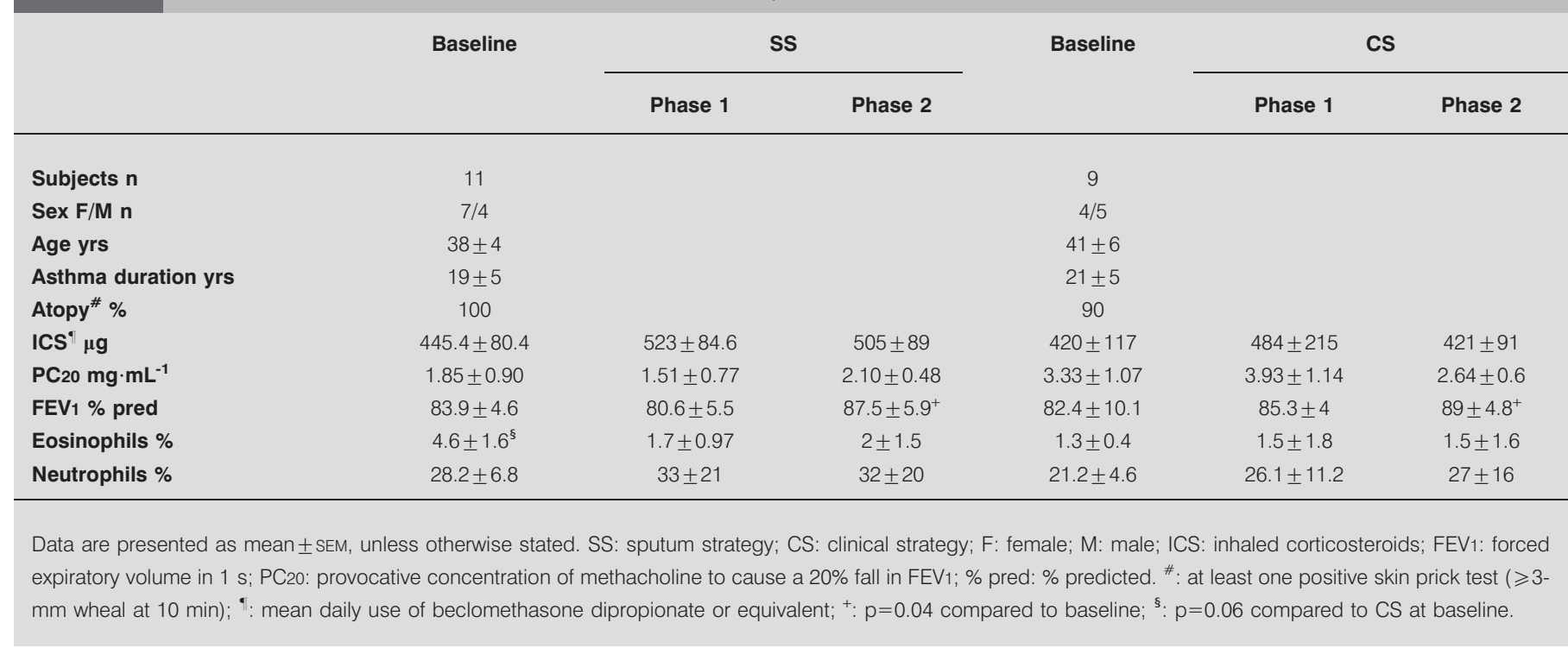


analysis was performed with interaction terms between the fixed factors. The unvaried normality assumptions were verified with the Shapiro-Wilk test, and Brown and Forsythe's variation of Levene's test statistics was used to verify the homogeneity of variances among treatments and among periods. The results were considered significant at $\mathrm{p} \leqslant 0.05$. All analyses were conducted using the statistical package SAS, version 9.2 (SAS Institute Inc, Cary, NC, USA).

\section{RESULTS}

All 20 subjects completed the 2-yr evaluation. These two groups of subjects had similar clinical characteristics (table 1). There were no significant differences in ICS doses taken by the two groups at the end of phase 1 and phase 2 . There was a significant increase in FEV1 in both groups at 2-yr follow-up $(p=0.04)$. Six of the 20 subjects had asthma exacerbations during phase 2: four in SS (two neutrophilic and two eosinophilic); and two in CS (neutrophilic).

We compared the percentage of sputum eosinophils in the two treatment strategies. Eosinophils tended to be higher in SS compared to CS $(\mathrm{p}=0.06)$ (table 1$)$ at baseline and were more variable $(p=0.04)$ during the duration of the study (mainly a result of the two eosinophilic exacerbations) in SS, but were similar at the end of phase 1 and after 2 yrs in both strategies (fig. 1). Neutrophil variation was similar in both strategies (fig. 1).

a)

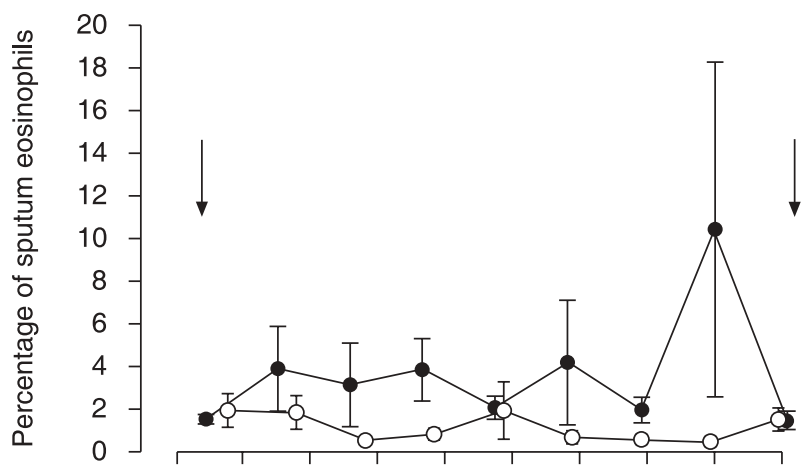

b)

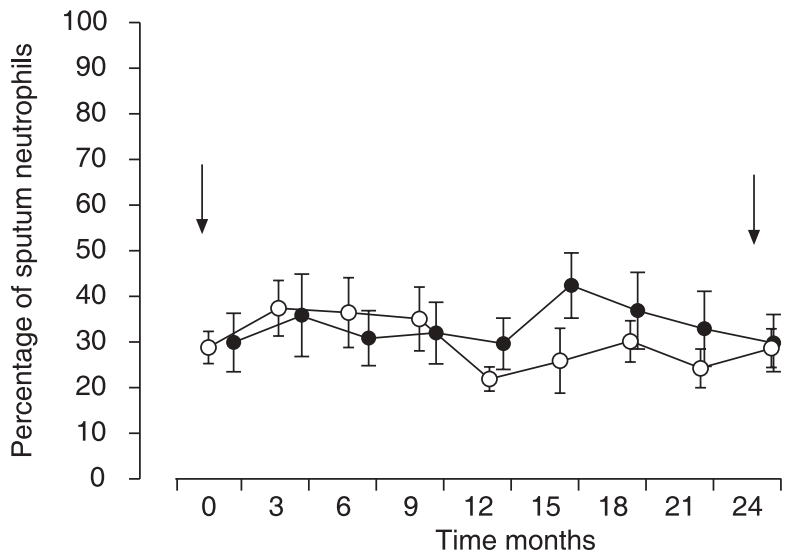

FIGURE 1. Variation in a) sputum eosinophils and b) neutrophils during the 24month follow-up in the sputum strategy $(\bullet)$ and clinical strategy $(\bigcirc)$ groups (arrows indicate bronchoscopy). Error bars represent SEM.
We examined cell counts in the bronchial biopsies. Bronchial mucosal CD3+ counts tended to be lower in CS compared with SS at the end of phase $1(165 \pm 18$ compared with $263 \pm 45$ per $\mathrm{mm}^{2} ; \mathrm{p}=0.08$ ) (fig. 2). These cells decreased significantly in SS at the end of phase 2 compared to CS $(\mathrm{p}=0.01)$. Total $(\mathrm{EG} 1+)$ and activated eosinophil (EG2+) cell counts were similar in both groups (fig. 2). However, after 2 yrs, total eosinophils decreased from $29 \pm 6$ to $10 \pm 4$ per $\mathrm{mm}^{2}(\mathrm{p}=0.014)$ in SS but did not change in CS (fig. 2). Activated eosinophils decreased significantly in both groups from $23 \pm 11$ to $8 \pm 4.2$ per $\mathrm{mm}^{2}$ (range from 0-97 to 0-48 per $\mathrm{mm}^{2}$ ) in SS (fig. 2), and from $17 \pm 1.3$ to $4 \pm 2.1$ per $\mathrm{mm}^{2}$ (range from $0-118$ to $0-20$ per $\mathrm{mm}^{2}$ ) in CS ( $p=0.041)$. Neutrophil and mast cell counts were similar at the end of phase 1 in both strategies (fig. 3). At 2-yr followup, neutrophils had decreased in CS from $78 \pm 16$ to $58 \pm 18$ per $\mathrm{mm}^{2}$ (range $14-151$ to $1-149$ per $\mathrm{mm}^{2} ; \mathrm{p}=0.03$ ) (fig. $3 \mathrm{~b}$ ) but not in SS (fig. 3a). No significant change was observed for tryptasepositive cell counts (fig. $3 \mathrm{c}$ and $\mathrm{d}$ ).

MUC5A expression was similar in CS and SS at the end of phase 1. At the end of phase 2, a significant decrease was observed in MUC5A staining in SS compared with CS $(\mathrm{p}=0.04)$ (fig. $4 a$ and $b$ ).

There were no significant correlations between the 2-yr changes in bronchial biopsy and sputum inflammatory cells in particular between the changes in eosinophil counts in the two compartments.

\section{Type I and type III collagen deposition}

We measured type I and III collagen deposition underneath the basement membrane in each group using techniques we previously reported [17]. There were no significant changes in the thickness of the type III and I collagen layers (fig. 5) below the basement membrane between CS and SS at baseline and these parameters did not change after 2 yrs of treatment.

\section{DISCUSSION}

In this pilot study, we showed that a strategy based on a closed evaluation of asthma control according to current guideline criteria, with or without the addition of the sputum eosinophil count to assess treatment needs, resulted in a reduction in activated eosinophils in bronchial tissue and a decrease in MUC5A expression. However, the thickness of the collagen below the basement membrane showed no significant change.

In a recent trial, we showed that a strategy based on the assessment of sputum eosinophilia as a guide to therapy resulted in a reduction in asthma exacerbations in moderate to severe asthmatic subjects [11]. There was no significant effect of this individually tailored treatment in subjects with mild asthma. In the context of this large study, we evaluated a subgroup of subjects in order to determine if there were any significant changes, not only in the sputum markers, but also in the airway wall markers of inflammation and remodelling. The present study included subjects with mostly mild asthma. At the end of the study, the two groups had a similar daily dose of ICS and similar sputum eosinophil counts. This is not surprising and was comparable to what was observed in the larger study. The success of the individually titrated treatment was because we accurately established the maintenance dose of treatment at the end of phase 1, and we adjusted treatment 

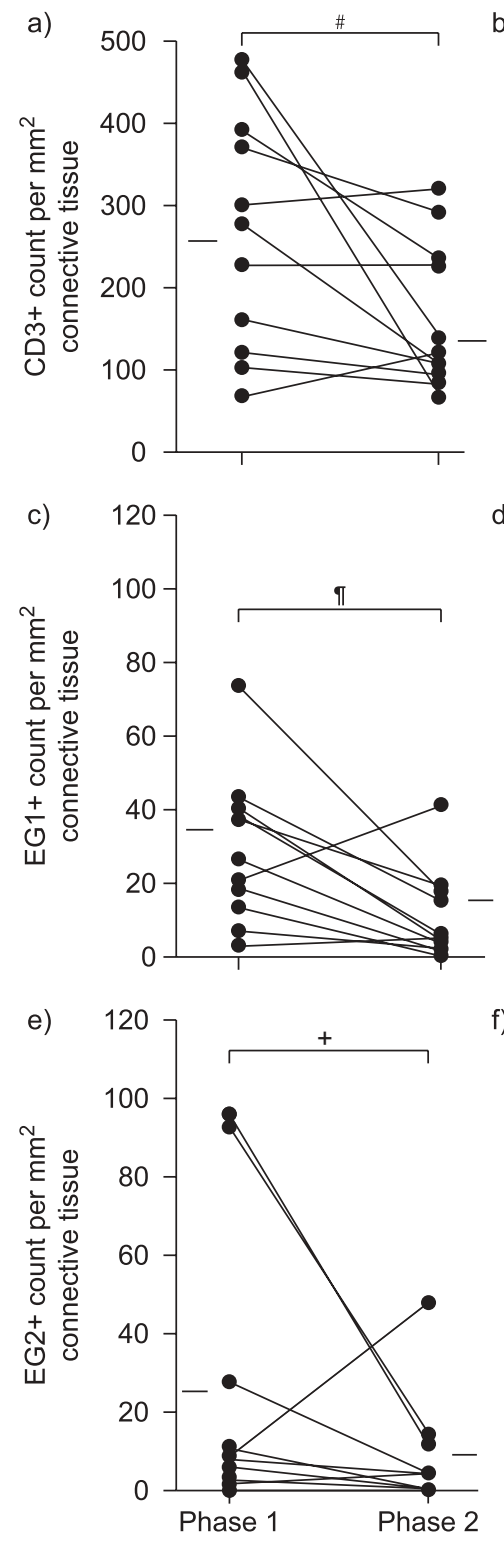

b)

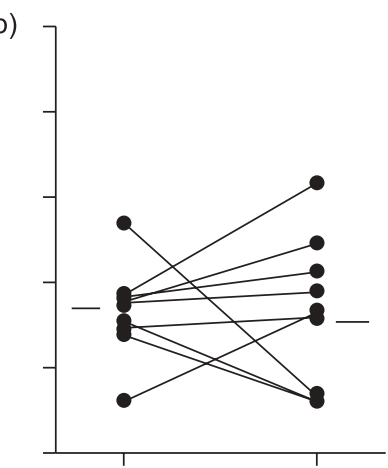

d)

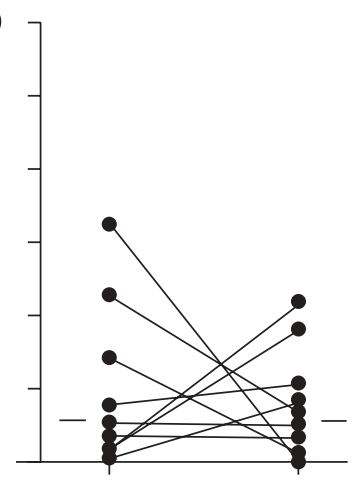

f)

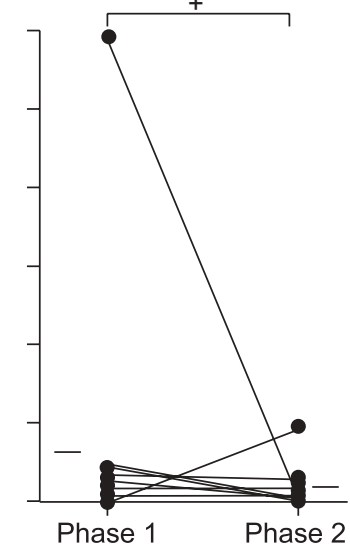

FIGURE 2. CD3+ cells ( $a$ and b), total eosinophils (EG1+) (c and d) and activated eosinophils (EG2+) (e and f) measured by immunostaining of bronchial biopsies in the sputum strategy ( $a, c$ and e) and clinical strategy (b, $d$ and f) groups. Mean values of positive cell counts per $\mathrm{mm}^{2}$ of connective tissue are shown. \#: $p=0.01 ;{ }^{\circ}: p=0.014 ;{ }^{+}: p=0.041$.

during each follow-up (by increasing ICS if the eosinophil levels were high). This prolonged the time to the first eosinophilic exacerbation in the sputum strategy. By appropriately treating the eosinophilic exacerbation using sputum cell counts, the subsequent (and, consequently, the total number of eosinophilic exacerbations) eosinophilic exacerbations were also decreased in the sputum strategy.

In the present study, management of asthma according to sputum eosinophilia or clinical criteria seems to have the same impact on tissue eosinophilia. There was a significant reduction in the number of activated eosinophils infiltrating the bronchial mucosa. This reduction may very well participate in the reduction in the release of inflammatory mediators by these
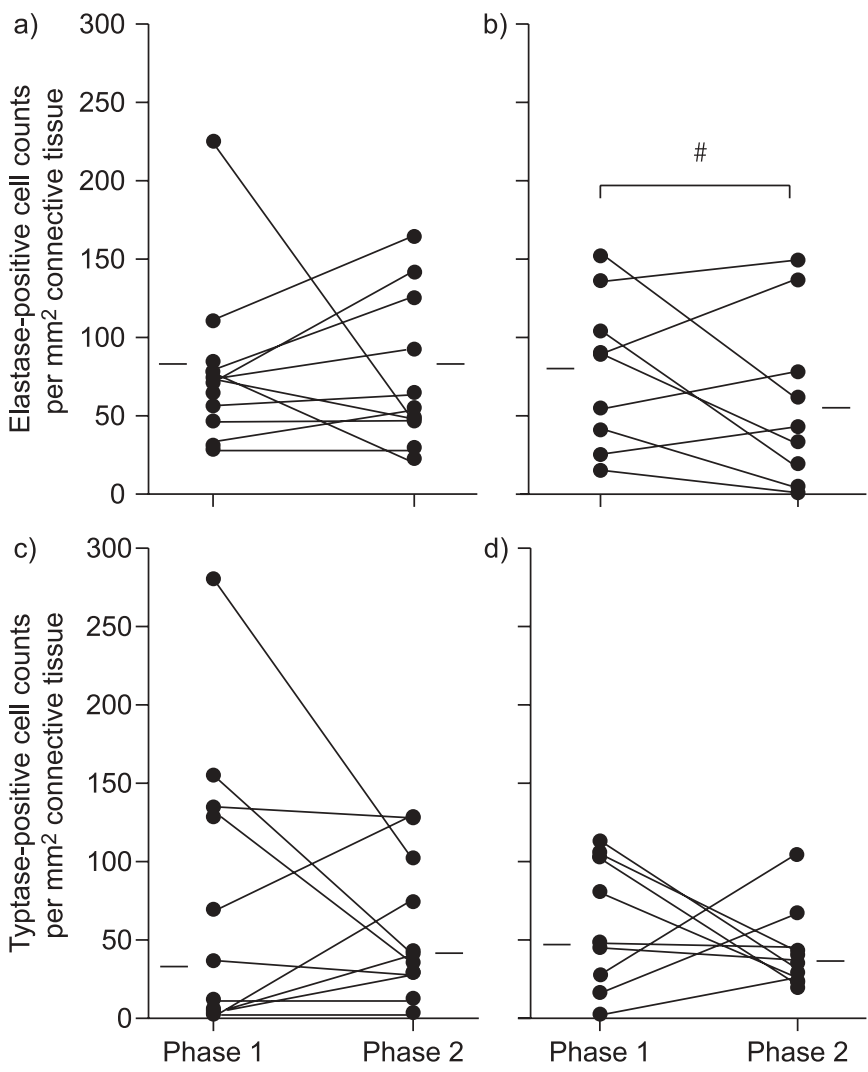

FIGURE 3. Neutrophil (elastase-positive cells) ( $a$ and b) and mast cell (tryptasepositive cells) ( $c$ and d) counts measured by immunostaining of bronchial biopsies in the sputum strategy ( $a$ and $c$ ) and clinical strategy (b and d) groups. Mean values of positive cell counts per $\mathrm{mm}^{2}$ of connective tissue are shown. ${ }^{\#}: p=0.03$.

cells and decrease inflammation in the bronchial mucosa. Interestingly, in the SS group, T-cell number in the bronchial tissue significantly decreased compared with in the CS group. However, at the end of phase 1, the number of T-cells in the bronchial mucosa of CS subjects tended to be lower than those of SS subjects. Thus, management of milder asthma according to sputum eosinophilia seems to have a similar efficacy to current clinical measures to control inflammation in the bronchial mucosa. With respect to asthma exacerbations, we observed similar neutrophilic exacerbations in both groups and two eosinophilic exacerbations in the SS group. However, both groups normalised their eosinophil and neutrophil counts before the second bronchoscopy.

We found no correlation between changes in bronchial biopsies and induced sputum inflammatory cells. These results are in agreement with the work of GROOTENDORST et al. [18], who compared inflammatory cell counts in induced sputum and bronchial biopsies in asthma. They found that there was no significant correlation between eosinophil and CD4+ T-cell counts in sputum and bronchial biopsies. This probably reflects the fact that induced sputum eosinophilia may represent a more short-term inflammatory state of the airways, often related to environmental exposures, whereas bronchial wall inflammation may represent a more long-term process.

Various new strategies have been recently proposed to determine treatment needs using noninvasive measures of 
a)

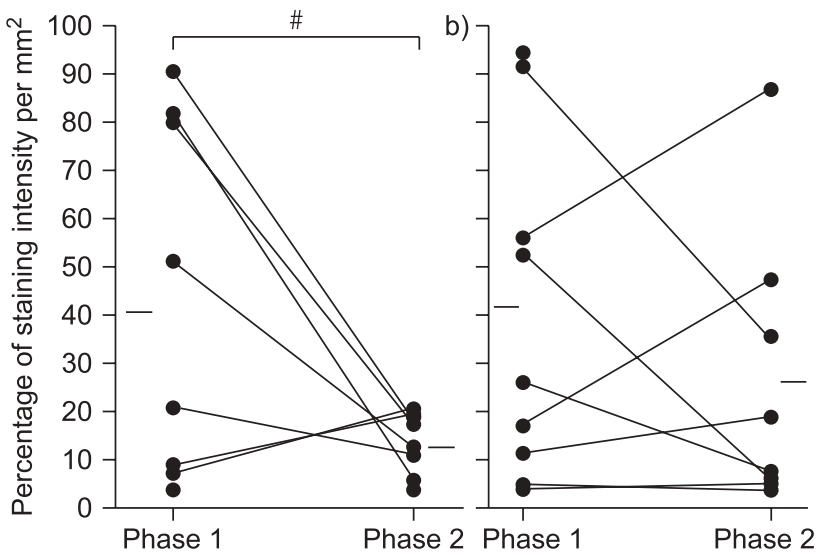

FIGURE 4. Mucin 5 A staining in the a) sputum strategy and b) clinical strategy groups. Mean values of percentage of staining intensity per $\mathrm{mm}^{2}$ are shown. \#: $p=0.03$.

airway inflammation, such as those based on exhaled nitric oxide and induced sputum analysis, in order to improve asthma monitoring and optimise the treatment of asthma [10, 19, 20]. Previously, GREEN et al. [9] have shown that a treatment strategy adjusting the dose of ICS to reduce airway eosinophilia resulted in better asthma control when compared to the usual British Thoracic Society guidelines. There were significantly fewer asthma exacerbations and hospital admissions with the strategy aiming at reducing airway inflammation [21]. A similar effect has been observed recently when using antiinterleukin-5 antibody treatment [19].

Although there is now good evidence that strategies targeting eosinophilic inflammation are beneficial in respect of asthma control, it is unclear whether this translates into beneficial effects on airway remodelling, a key feature of asthma. When looking at markers of remodelling, we found that mucin expression, a key feature of asthma, is reduced in SS but not in CS, suggesting that optimal control of eosinophilic inflammation either directly reduces mucus secretion, or is a marker of a wider effect on the mechanisms regulating this process. These mechanisms are complex and involve cytokines produced by T-cells such as interleukin (IL)-13 [22]. Elucidation of mechanisms whereby MUC5A staining is reduced by corticosteroids is beyond the scope of the current study, but it is worthy of note that in the SS group we observed a significant decrease in Tcell count.

We did not observe any change in collagen I and III deposition underneath the epithelial layer over the 2-yr period. These features of remodelling have been shown consistently to be characteristic of asthma [23] that is resistant to corticosteroid treatment [24]. A notable exception is the data from the study by SONT et al. [7], who found that using a treatment strategy targeting bronchial hyperresponsiveness as a guide to the dose of ICS reduced the subepithelial collagen layer.

The results of this study have to be interpreted with caution, as the size of the sample was small. To observe changes in subepithelial collagen deposition, a much larger number of patients is needed. Furthermore, based on our data and previous studies [25, 26], higher doses given for longer periods
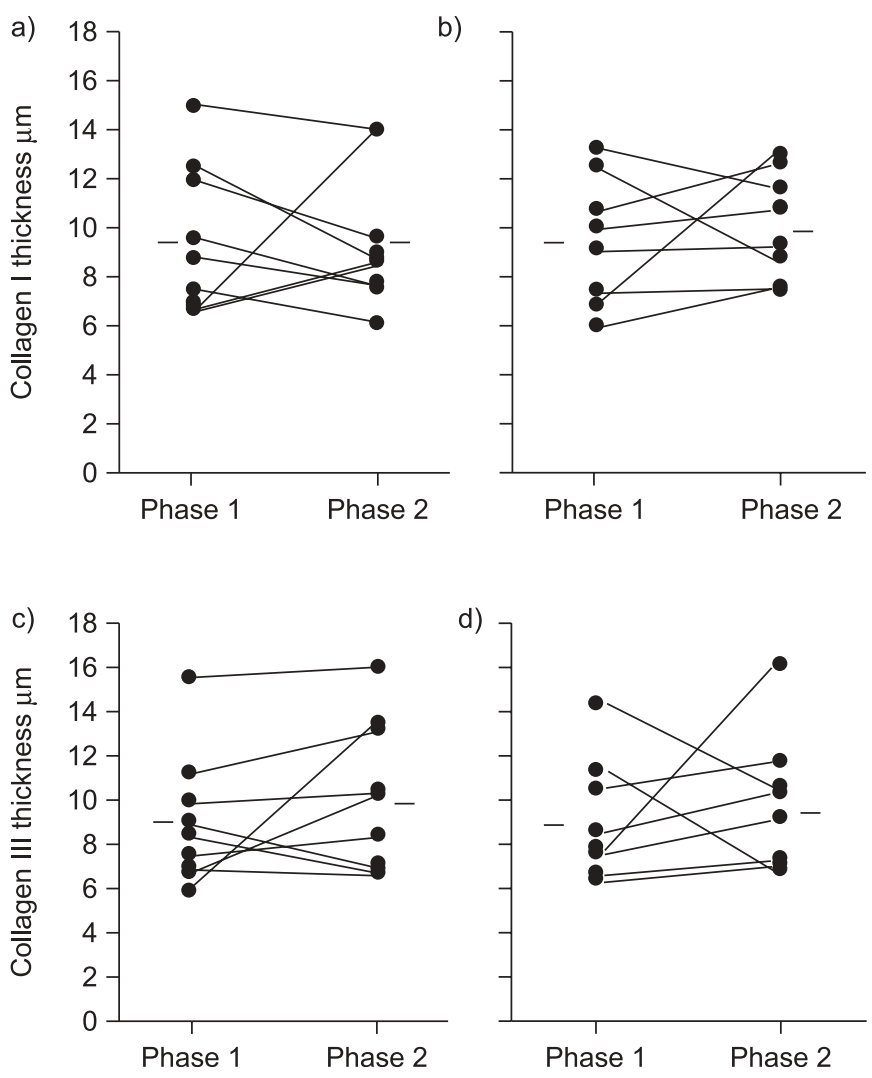

FIGURE 5. Sub-epithelial thickness of type I and III collagens in the sputum strategy (a and c) and clinical strategy (b and d) groups.

would probably be needed to observe significant changes. In the study by SONT et al. [7], the reduction of AHR was achieved at the expense of doubling of the dose of ICS. In our study, both groups received similar doses of ICS during 24 months of follow-up, and were lower than those used by SONT et al. [7]. In support of this explanation, WARD et al. [8] reported significant, although limited, changes in airway wall fibrosis with high dose of ICS for 52 weeks, whereas the other parameters improved more rapidly.

The benefits of reducing airway remodelling may be to reduce long-term decline of pulmonary function or to reduce the severity of disease, although this is still controversial. Monitoring airway eosinophilia may at least reduce exacerbations and improve asthma control, as shown by GREEN et al. [9] and others, whereas the beneficial effects of acting on remodelling have to be further studied before recommending a strategy aimed at reducing this process. It is possible that the strategy used changed other components of extracellular matrix that we did not measure, such as the content of proteoglycans. Recently, we showed that mast cells stimulate bronchial fibroblasts obtained from asthmatic patients, but not from normal subjects, to produce procollagen through IL-4 production [27]. In the present study, we found no significant changes in mast cell count over the 2-yr follow-up. WARD et al. [28] previously reported that variation in subepithelial fibrosis was related to variations in the percentage of mast cells in the bronchoalveolar lavage and suggested that mast cells might be associated with the development of airway remodelling. 
However, the ICS doses used in our study, determined by asthma control level instead of arbitrary dosing, may not be sufficient to observe a change in these parameters.

In conclusion, this study reports important data on the comparative influence of clinical and induced sputum strategies for the assessment of medication needs in mild to moderate asthma. It showed a significant reduction in some markers of inflammation and mucin production, but no change in subepithelial collagen deposition. It would be of interest to further study the influence of these strategies in patients with higher baseline eosinophil levels and more severe disease, or earlier in the course of the disease. Finally, it might be that the best strategy will be one in which eosinophilia is used as a target to reduce the exacerbation rate, whereas hyperresponsiveness is used as a target aimed at reversing airway remodelling.

\section{SUPPORT STATEMENT}

This work was supported by the Canadian Institutes for Health Research (Ottawa, ON, Canada). J. Chakir is the recipient of Chercheur-Boursier Awards from the Fonds de la Recherche en Santé du Québec (Montreal, QC, Canada). P. Nair is supported by a Canada Research Chair in Airway Inflammation.

\section{STATEMENT OF INTEREST}

None declared.

\section{ACKNOWLEDGEMENTS}

We thank all subjects for their participation in this study. We are grateful to S. Simard (Institut Universitaire de Cardiologie et de Pneumologie de Québec, Québec, QC, Canada) for his help with statistical analysis. We thank la banque de tissue du Réseau en santé respiratoire du FRSQ (Fonds de la Recherche en Santé du Québec, Montreal, QC, Canada).

\section{REFERENCES}

1 Hargreave FE, Leigh R, Parameswaran K. Asthma as a disease concept. Lancet 2006; 368: 1415-1416.

2 Elston WJ, Whittaker AJ, Khan LN, et al. Safety of research bronchoscopy, biopsy and bronchoalveolar lavage in asthma. Eur Respir J 2004; 24: 375-377.

3 Jeffery P, Holgate S, Wenzel S. Methods for the assessment of endobronchial biopsies in clinical research: application to studies of pathogenesis and the effects of treatment. Am J Respir Crit Care Med 2003; 168: S1-S17.

4 Chanez P, Bourdin A, Vachier I, et al. Effects of inhaled corticosteroids on pathology in asthma and chronic obstructive pulmonary disease. Proc Am Thorac Soc 2004; 1: 184-190.

5 Boulet LP, Chakir J, Milot J, et al. Effect of salmeterol on allergeninduced airway inflammation in mild allergic asthma. Clin Exp Allergy 2001; 31: 430-437.

6 Boulet LP, Turcotte H, Laviolette M, et al. Airway hyperresponsiveness, inflammation, and subepithelial collagen deposition in recently diagnosed versus long-standing mild asthma. Influence of inhaled corticosteroids. Am J Respir Crit Care Med 2000; 162: 1308-1313.

7 Sont JK, Willems LN, Bel EH, et al. Clinical control and histopathologic outcome of asthma when using airway hyperresponsiveness as an additional guide to long-term treatment. The AMPUL Study Group. Am J Respir Crit Care Med 1999; 159: 1043-1051.

8 Ward C, Pais M, Bish R, et al. Airway inflammation, basement membrane thickening and bronchial hyperresponsiveness in asthma. Thorax 2002; 57: 309-316.
9 Green RH, Brightling CE, McKenna S, et al. Asthma exacerbations and sputum eosinophil counts: a randomised controlled trial. Lancet 2002; 360: 1715-1721.

10 Zacharasiewicz A, Wilson N, Lex C, et al. Clinical use of noninvasive measurements of airway inflammation in steroid reduction in children. Am J Respir Crit Care Med 2005; 171: 1077-1082.

11 Jayaram L, Pizzichini MM, Cook RJ, et al. Determining asthma treatment by monitoring sputum cell counts: effect on exacerbations. Eur Respir J 2006; 27: 483-494.

12 Lemiere C, Bai T, Balter $\mathrm{M}$, et al. Adult Asthma Consensus Guidelines Update 2003. Can Respir J 2004; 11: 9A-18A.

13 Pizzichini E, Pizzichini MM, Efthimiadis A, et al. Indices of airway inflammation in induced sputum: reproducibility and validity of cell and fluid-phase measurements. Am J Respir Crit Care Med 1996; 154: 308-317.

14 Chakir J, Hamid Q, Bossé M, et al. Bronchial inflammation in corticosteroid-sensitive and corticosteroid-resistant asthma at baseline and on oral corticosteroid treatment. Clin Exp Allergy 2002; 32: 578-582.

15 Loubaki L, Jacques E, Semlali A, et al. Tumor necrosis factor- $\alpha$ expression in uvular tissues differs between snorers and apneic patients. Chest 2008; 134: 911-918.

16 Series F, Chakir J, Boivin D. Influence of weight and sleep apnea status on immunologic and structural features of the uvula. Am J Respir Crit Care Med 2004; 170: 1114-1119.

17 Chakir J, Laviolette $\mathrm{M}$, Boutet $\mathrm{M}$, et al. Lower airways remodeling in nonasthmatic subjects with allergic rhinitis. Lab Invest 1996; 75: 735-744.

18 Grootendorst DC, Sont JK, Willems LN, et al. Comparison of inflammatory cell counts in asthma: induced sputum versus bronchoalveolar lavage and bronchial biopsies. Clin Exp Allergy 1997; 27: 769-779.

19 Nair P, Pizzichini MM, Kjarsgaard M, et al. Mepolizumab for prednisone-dependent asthma with sputum eosinophilia. N Engl J Med 2009; 360: 985-993.

20 Jones SL, Herbison P, Cowan JO, et al. Exhaled NO and assessment of anti-inflammatory effects of inhaled steroid: dose-response relationship. Eur Respir J 2002; 20: 601-608

21 in't Veen JC, Smits HH, Hiemstra PS, et al. Lung function and sputum characteristics of patients with severe asthma during an induced exacerbation by double-blind steroid withdrawal. Am J Respir Crit Care Med 1999; 160: 93-99.

22 Zhen G, Park SW, Nguyenvu LT, et al. IL-13 and epidermal growth factor receptor have critical but distinct roles in epithelial cell mucin production. Am J Respir Cell Mol Biol 2007; 36: 244-253.

23 Bousquet J, Jeffery PK, Busse WW, et al. Asthma. From bronchoconstriction to airways inflammation and remodeling. Am J Respir Crit Care Med 2000; 161: 1720-1745.

24 Benayoun L, Druilhe A, Dombret MC, et al. Airway structural alterations selectively associated with severe asthma. Am J Respir Crit Care Med 2003; 167: 1360-1368.

25 Boulet LP, Turcotte H, Laviolette M, et al. Airway hyperresponsiveness, inflammation, and subepithelial collagen deposition in recently diagnosed versus long-standing mild asthma. Influence of inhaled corticosteroids. Am J Respir Crit Care Med 2000; 162: 1308-1313.

26 Ward C, Pais M, Bish R, et al. Airway inflammation, basement membrane thickening and bronchial hyperresponsiveness in asthma. Thorax 2002; 57: 309-316.

27 Plante S, Semlali A, Joubert $\mathrm{P}$, et al. Mast cells regulate procollagen I (alpha 1) production by bronchial fibroblasts derived from subjects with asthma through IL-4/IL-4 delta 2 ratio. J Allergy Clin Immunol 2006; 117: 1321-1327.

28 Ward C, Reid DW, Orsida BE, et al. Inter-relationships between airway inflammation, reticular basement membrane thickening and bronchial hyper-reactivity to methacholine in asthma; a systematic bronchoalveolar lavage and airway biopsy analysis Clin Exp Allergy 2005; 35: 1565-1571. 